\title{
Feasibility and safety of bedside percutaneous biliary drainage in patients with severe cholangitis
}

\author{
Pankaj Gupta ${ }^{1} @$ - Muniraju Maralakunte ${ }^{1}$. Naveen Kalra ${ }^{1}$. Jayanta Samanta ${ }^{2} \cdot$ Vishal Sharma $^{2}$. \\ Harshal Mandavdhare ${ }^{2} \cdot$ Usha Dutta ${ }^{2} \cdot$ Rakesh Kochhar $^{2} \cdot$ Manavjit Singh Sandhu $^{1}$
}

Received: 27 August 2020 / Revised: 3 October 2020 / Accepted: 10 October 2020 / Published online: 23 October 2020

(c) Springer Science+Business Media, LLC, part of Springer Nature 2020

\begin{abstract}
Purpose To evaluate the safety and feasibility of bedside percutaneous transhepatic biliary drainage (PTBD) as a salvage procedure in patients with severe cholangitis in the intensive care unit (ICU).

Methods This retrospective study evaluated records of consecutive patients with severe cholangitis who were admitted in the ICU. Bedside PTBD was performed using ultrasound guidance. The level and cause of biliary obstruction were recorded. The technical success of the procedure and complications were recorded.

Results Ten patients (six males, mean age 53.8 years) underwent bedside PTBD. Six patients had distal common bile duct blockade [periampullary carcinoma $(n=2)$, carcinoma pancreas $(n=2)$, choledocholithiasis $(n=1)$, and benign stricture $(n=1)$ ]. Four patients had malignant hilar stricture [cholangiocarcinoma $(n=3)$ and carcinoma gallbladder $(n=1)]$. Technical success was achieved in all. One patient underwent bilateral PTBD. Left and right PTBD were performed in 5 and 4 patients, respectively. There were no major complications. Transient hemobilia occurred in two patients.

Conclusion Bedside PTBD is safe and technically feasible. Prospective studies are required to establish this procedure into routine clinical practice.
\end{abstract}

Keywords Biliary drainage $\cdot$ Percutaneous $\cdot$ Intensive care unit

\section{Introduction}

The advent of advanced endoscopic techniques has reduced the utilization of percutaneous transhepatic biliary drainage (PTBD). Yet, there is an essence and scope for PTBD in the setting of hilar strictures, failed endoscopic retrograde cholangiopancreatography (ERCP), or altered anatomy after the entero-biliary surgical procedures $[1,2]$. The most crucial indication where PTBD might be a life-saving procedure is severe cholangitis [3]. Besides, the medical measures, the essential component of management is the relief of biliary obstruction. In patients with severe cholangitis, intensive care unit (ICU) management may be necessary. Many patients with severe cholangitis may be on mechanical

Pankaj Gupta

Pankajgupta959@gmail.com

1 Department of Radiodiagnosis and Imaging, PGIMER, Chandigarh, India

2 Department of Gastroenterology, PGIMER, Chandigarh, India ventilation. Biliary drainage in this group of patients poses a challenge. Though bedside ERCP has been reported, the facility and expertise are not available in most centers. Shifting these patients to the ERCP suite may pose a risk. These factors cause a delay in biliary drainage and worsening of the sepsis. PTBD is an effective minimally invasive interventional radiology procedure for draining the biliary system in the setting of cholangitis. Bedside PTBD will allow rapid decompression of the biliary system and may act as a salvage procedure [4-6]. The present study aims to evaluate the feasibility and safety of bedside PTBD in the management of patients with severe cholangitis.

\section{Methods and materials}

Records of consecutive bedside PTBD procedures performed on the patients with severe cholangitis receiving ICU care, between November 2017 and December 2019, were evaluated. Only patients who could not be shifted to the interventional radiology (IR) suite for fluoroscopic guidance 
were included. The failure to mobilize the patients outside the ICU was either because of unstable condition. The latter group of patients, who were not on mechanical ventilation at the time of PTBD, had impending need for mechanical ventilation or need for escalation of ionotropic support, as assessed by the attending gastroenterologist, that precluded their shifting to the IR suite. Tokyo guidelines 2018 were utilized to diagnose as well as to assess the severity of the acute cholangitis in subjects with obstructive biliopathy [7]. Preprocedural assessment included the review of the latest cross-sectional imaging and coagulation profile (Fig. 1). Level of biliary obstruction was assessed. Proximal obstruction were defined as those at the hilum (above the level of cystic duct orifice) and distal obstructions as those that involved bile ducts at or below the level of cystic duct orifice [8].The PTBD procedures were performed by an interventional radiologist with five years of experience in the abdominal non-vascular interventions. Assistance was provided by a radiology resident. The procedures were performed using only ultrasound (US) guidance. The procedural notes included the route (left vs. right) of entry, technical success (defined as the ability to achieve drainage as planned), complications, and short-term follow-up (defined as course in the hospital) were recorded. In our previous study on the PTBD in patients at risk for adverse events, we reported bedside PTBD in 5 patients [3]. The data of those patients are also included in this study.

\section{Technique}

Analgesia was provided with slow intravenous injection of tramadol $100 \mathrm{mg}$, supplemented with intravenous antiemetic, Ondansetron $4 \mathrm{mg}$ for those who were not on mechanical ventilation. The patient's upper abdomen was prepared (10\% betadine) and draped. Local anesthetic was administered at the puncture site using $10 \mathrm{~mL}$ of $2 \%$ of lidocaine. A suitable duct was chosen after a detailed assessment with sonography. Color flow and Doppler was used to differentiate between bile ducts and vessels. The entire biliary system was examined by US with the help 3.5-5 MHz frequency transducer. Since these patients were intubated, no breath-hold instruction was given. The $18 \mathrm{G} / 22 \mathrm{G}$ needle was employed for puncture of the bile duct under sonographic guidance, and tip position within the duct was visualized on the greyscale tissue harmonic imaging. After removal of the stylet, the correct position of the
Fig. 1 Axial (a) and coronal (b) CT images in an 85-year-old male with hilar cholangiocarcinoma shows multiple cholangitis abscesses in the right lobe of liver (arrows, $\mathbf{a}$ and $\mathbf{b}$ ). There is mild right and significant left biliary dilatation (long arrows, b). Ultrasound image (c) bilobar intrahepatic biliary dilatation (arrow) and the stricture (short arrow). Ultrasound image (d) shows the PTBD in the left duct (arrow)

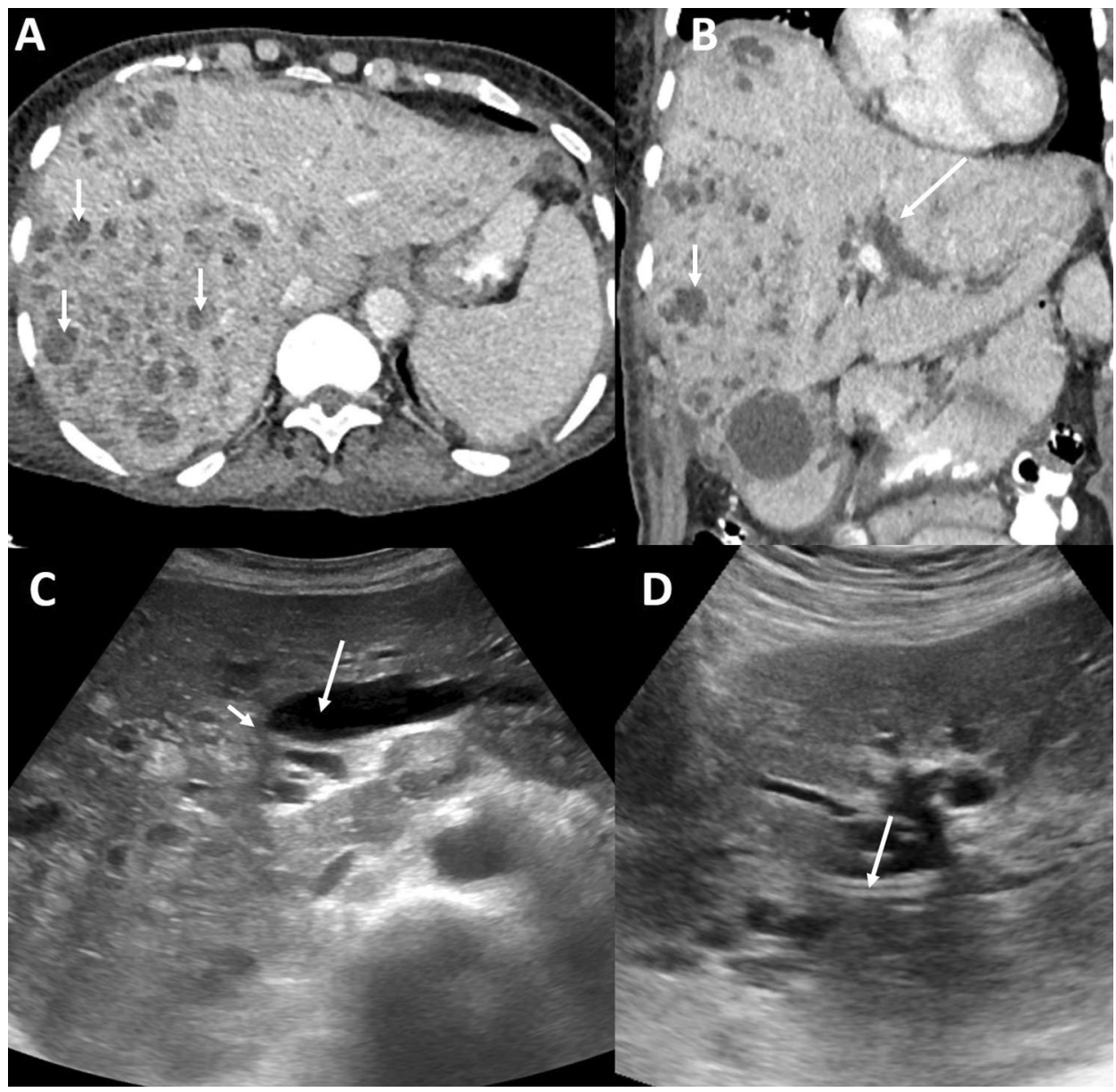


needle tip was ascertained by the free flow of bile through the needle. Following this, a hydrophilic guidewire was manipulated into the biliary system through the needle under real-time sonographic control. Stiff fascial dilators (ranging 6-8 F) were utilized for tract dilatation after removal of the needle over the guidewire. Subsequently, biliary drainage catheter $(6-8 \mathrm{~F})$ was advanced over the guidewire and left in the accessed duct. Since all subjects were on antibiotics, no additional antibiotics were administered. Post-procedure assessment was performed with bedside US to assess the decompression of the bile ducts, as well as to assess for procedure-related complications, including perihepatic fluid, and status of the vessels. The nature and amount of drain output was also assessed.

\section{Results}

A total of ten patients underwent bedside PTBD procedures during the study period. There were seven males and three females. The mean age was 53.8 years (age range, $27-85$ years). All the patients had severe sepsis and shock with associated multiorgan failure. Five patients were receiving mechanical ventilation.

On imaging, all the patients had extrahepatic biliary obstruction with different levels of block. Six patients had distal obstruction due to the periampullary carcinoma and carcinoma head of pancreas in two patients each and choledocholithiasis and benign stricture in one patient each. Four patients had malignant hilar stricture (proximal obstruction) due to cholangiocarcinoma in three and carcinoma gallbladder in one.

Technical success was achieved in all patients. A total of 11 PTBD catheters (two $6 \mathrm{~F}$, one $7 \mathrm{~F}$, and eight $8 \mathrm{~F}$ catheters) were utilized. One patient underwent bilateral PTBD. Five patients had left PTBD, and four patients had right PTBD. Initially, all the patients had external drainage catheters, and later two patients who were discharged from the ICU underwent internalization. Other two patients who were discharged from the ICU were still on external drainage at the time of last follow-up. Six patients died due to pre-existing multiorgan failure. There were no major complications. Minor complications in the form of transient hemobilia were seen in 2 patients. However, there were liver hemorrhagic complications on follow-up US and Doppler. The demographic, clinical details, imaging, procedural details, procedural endpoints, and outcomes of the subjects are summarized in Table1.

\section{Discussion}

Malignant biliary obstruction is a common condition presenting to the medical and surgical specialties. The common causes include cholangiocarcinoma, pancreatic cancer, metastatic disease, and carcinoma gallbladder [2]. Carcinoma gallbladder particularly has a poor prognosis $[9$, 10]. Severe cholangitis occurs as a complication of biliary obstruction from both benign and malignant conditions [1]. It is one of the most dreaded gastrointestinal emergencies [11]. If not managed timely, patients rapidly go on to develop multisystem organ failure with resultant high mortality. Besides, medical measures and organ support, the cornerstone of management is urgent biliary drainage. In the setting of severe cholangitis with the need for continuous organ support and monitoring, conventional fluoroscopyguided ERCP or PTBD might not be feasible. Supportive medications include inotropes, ventilator support and multiple tubes and lines. Practically, it is not a simple task to shift these patients and their connected tubes/lines along with the supportive equipment into the interventional radiology suite. Few case reports describing X-ray free ERCP have been published [4-6]. US-guided PTBD by an interventional radiologist may be an alternative. However, there is no published literature reporting the safety and feasibility of bedside PTBD. The present study reports the technical outcomes in a series of ten patients with severe cholangitis who underwent bedside PTBD as a salvage procedure. PTBD was technically successful in all patients. There were no major complications or mortality related to the procedure. Six patients died despite bedside PTBD. This is related to the multisystem organ failure and refractory shock that was present at the time of the procedure. This fact also highlights that drainage should be performed early in the course of the severe cholangitis.

Though, none of the published studies have specifically reported a bedside PTBD in patients with severe cholangitis, US access to the bile ducts has been reported previously. The use of Doppler allows safe entry into the bile ducts. Koito et al. initially described the use of Doppler during US-PTBD [11]. Later in their study of 30 patients, they successfully placed PTBD under US guidance with the use of Doppler in all patients with no major complications [12]. Another study reported technical success of US-guided PTBD comparable to fluoroscopically guided PTBD (F-PTBD) [13]. However, in this study, all F-PTBDs were right sided, and US-PTBDs were left sided. Major complications were encountered only in the F-PTBD group. Successful US-guided PTBD has been reported even for the non-dilated system. In a study by Shimizu et al., PTBD was technically successful in 33/35 patients with the non-dilated system using a peripheral portal vein-oriented bile duct puncture [14]. We had two patients with minimally dilated ducts in whom PTBD was successfully placed under US guidance.

There were a few limitations to our study. All the procedures were performed by a single interventional radiologist. The co-morbidities, number of organ failure, and timing of PTBD with relation to onset of organ failure were not 


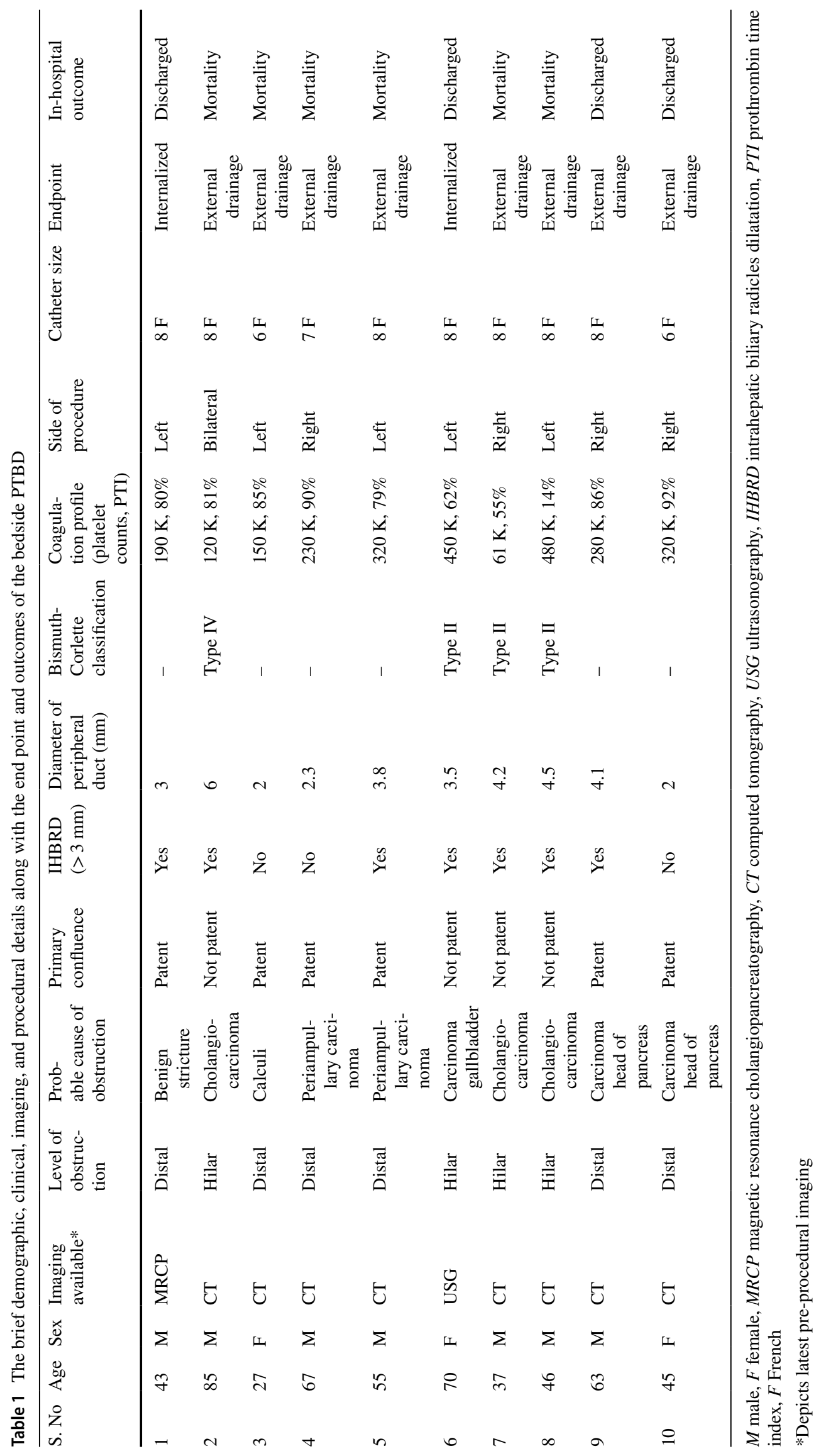


recorded. All these factors are important determinants of mortality in the ICU setting. The follow-up was limited to the hospital course only.

In conclusion, the present study reported the feasibility and safety of the bedside PTBD as a salvage procedure in the ICU setting. However, a more extensive prospective study with appropriate timing of the procedure and accounting for the co-morbid conditions will demonstrate the clinical effectiveness of this procedure.

\section{Compliance with ethical standards}

Conflict of interest The authors report no conflict of interest or financial disclosure.

\section{References}

1. Funaki B. Percutaneous Biliary Drainage. Semin Intervent Radiol. 2007;24(2):268-271.

2. Gupta P, Gupta J, Kumar MP. Imaging in obstructive jaundice: what a radiologist needs to know before doing a percutaneous transhepatic biliary drainage. J Clinical Radiol ISVIR 2020;4:31-37.

3. Gupta P, Maralakunte M, Rathee S, et al. Percutaneous transhepatic biliary drainage in patients at higher risk for adverse events: experience from a tertiary care referral center. Abdom Radiol. 2019;45(8):2457-553.

4. Sreenivas DV, Kumar A. Urgent bedside endoscopic nasobiliary drainage without fluoroscopic monitoring. Hepatogastroenterology 1998;45(24):2042-3.

5. Chawla YK, Sharma BC, Singh R, Sharma TR, Dilawari JB. Emergency endoscopic nasobiliary drainage without the aid of fluoroscopy. Indian J Gastroenterol. 1993;12(3):97-8.
6. Shah R, Qayed E. Bedside Endoscopic Retrograde Cholangiopancreatography Using Portable X-Ray in Acute Severe Cholangitis. Case Rep Gastrointest Med. 2018;2018:8763671.

7. Miura F, Okamoto K, Takada T, et al. Tokyo Guidelines 2018: initial management of acute biliary infection and flowchart for acute cholangitis. J Hepatobiliary Pancreat Sci. 2018;25:31-40

8. Chen JH, Sun CK, Liao CS, Chua CS. Self-expandable metallic stents for malignant biliary obstruction: efficacy on proximal and distal tumors. World J Gastroenterol. 2006;12(1):119-22.

9. Gupta P, Kumar M, Sharma V, Dutta U, Sandhu MS. Evaluation of gallbladder wall thickening: a multimodality imaging approach. Exp Rev Gastroenterol Hepatol 2020;463-473.

10. Gupta P, Meghashyam K, Marodia Y, et al. Locally advanced gallbladder cancer: a review of the criteria and role of imaging. Abdom Radiol (NY). 2020 Sep 18. https://doi.org/10.1007/s0026 1-020-02756-4. Epub ahead of print. PMID: 32945922.

11. Ralls PW, Mayekawa OS, Lee KP, et al: The use of color Doppler sonography to distinguish dilated intrahepatic ducts from vascular structures. Am J Roentgenol 1998;152:291

12. Koito K, Namieno T, Nagakawa T, Morita K. Percutaneous transhepatic biliary drainage using color Doppler ultrasonography. J Ultrasound Med. 1996;15(3):203-6.

13. Nennstiel S, Treiber M, Faber A, Haller B, von Delius S, Schmid RM, Neu B. Comparison of Ultrasound and Fluoroscopically Guided Percutaneous Transhepatic Biliary Drainage. Dig Dis. 2019;37(1):77-86.

14. Shimizu H, Kato A, Takayashiki T, et al. Peripheral portal vein-oriented non-dilated bile duct puncture for percutaneous transhepatic biliary drainage. World J Gastroenterol. 2015;21(44):12628-34.

Publisher's Note Springer Nature remains neutral with regard to jurisdictional claims in published maps and institutional affiliations. 